\title{
Absent Pulmonary Valve Syndrome with Left Hemitruncus
}

\author{
Ashraf M. Aly, M.D., Ph.D. ${ }^{1}$ Deborah A. Reid, R.D.M.S. ${ }^{2}$ Amyn K. Jiwani, M.D. ${ }^{1}$ Pooja H. Desai, M.D. ${ }^{1}$ \\ 1 Division of Pediatric Cardiology, Department of Pediatrics, University \\ of Texas Medical Branch-Galveston, Galveston, Texas \\ 2 Division of Maternal Fetal Medicine, Department of Obstetrics and \\ Gynecology, University of Texas Medical Branch-Galveston, \\ Galveston, Texas \\ Address for correspondence and reprint requests Ashraf M. Aly, M.D., \\ Ph.D., Professor of Pediatrics, Director, Division of Pediatric \\ Cardiology, Department of Pediatrics, The University of Texas Medical \\ Branch, 301 University Blvd., Galveston, TX 77555-0367 \\ (e-mail: amaly@utmb.edu).
}

Am J Perinatol Rep 2012;2:51-54.

\begin{abstract}
Keywords

- tetralogy of Fallot

- absent pulmonary valve

- left hemitruncus

- fetal echocardiography

Absent pulmonary valve syndrome (APVS) is a rare congenital heart defect, usually associated with tetralogy of Fallot (TOF), although other associations have been described. A pregnant woman was referred to fetal echocardiography clinic from the Maternal Fetal Medicine department due to abnormal findings on routine antenatal ultrasound, showing a pulsatile cystic mass above the left atrium and a suspected TOF. A fetal echocardiogram confirmed the presence of TOF/APVS. The pulsatile cystic mass was the aneurysmally dilated main pulmonary artery. The exact origin of the left pulmonary artery (LPA) was not clearly established prenatally. A postnatal echocardiogram of the neonate showed an abnormal origin of the LPA from the ascending aorta (hemitruncus). The neonate subsequently underwent surgical repair with a good outcome. We present a novel case of a TOF/APVS associated with an abnormal origin of the LPA from the ascending aorta.
\end{abstract}

Absent pulmonary valve syndrome (APVS) has been reported in 3 to $6 \%$ of all cases of tetralogy of Fallot (TOF), and the incidence is even higher in the prenatal population due to high rate of fetal demise. ${ }^{1}$ Although TOF is the most commonly associated congenital heart defect (CHD) in APVS, other defects have been described. ${ }^{2,3}$ Absence of a patent ductus arteriosus (PDA) has been postulated as a cause of APVS. ${ }^{4}$ The pulmonary valve leaflets are either rudimentary or completely absent, and there is pulmonary valve stenosis and incompetence. The pulmonary arteries are usually dilated and are often aneurysmal in size. This frequently causes compression of bronchial tree and the development of bronchomalacia, which explains the associated respiratory symptoms of this condition.

\section{Case Report}

A 23-year-old pregnant Hispanic woman, gravida 2, para 1, was referred to pediatric cardiology at 22 weeks' gestation by

received

January 4, 2012

accepted after revision

March 3, 2012

published online

July 16, 2012

the Maternal Fetal Medicine department for a fetal echocardiography and counseling. Antenatal ultrasound showed a pulsating cystic mass above the left atrium and a suspected TOF. The patient had a negative family history for chromosomal abnormalities, CHDs, or unexpected cardiac death at a young age. She denied use of drugs, alcohol, tobacco smoking, or exposure to toxic chemicals.

A fetal echocardiogram showed a four-chamber heart with a ventricular septal defect (VSD) and an overriding aorta (-Fig. 1). There was hypoplasia of the pulmonary valve annulus with complete absence of the pulmonary valve leaflets (-Fig. 2). The main pulmonary artery (MPA) and the right pulmonary artery (RPA) were markedly dilated. The origin of the left pulmonary artery (LPA) was not clearly established but did not seem to connect to the MPA. No ductus arteriosus was seen. Pulsed wave Doppler/color Doppler evaluation showed pulmonary valve stenosis and regurgitation.

The patient was counseled regarding the association of these findings with chromosomal abnormalities and other

Copyright $\odot 2012$ by Thieme Medical Publishers, Inc., 333 Seventh Avenue, New York, NY 10001, USA. Tel: +1(212) 584-4662.
DOI http://dx.doi.org/ $10.1055 / \mathrm{s}-0032-1316464$. ISSN 2157-6998. 


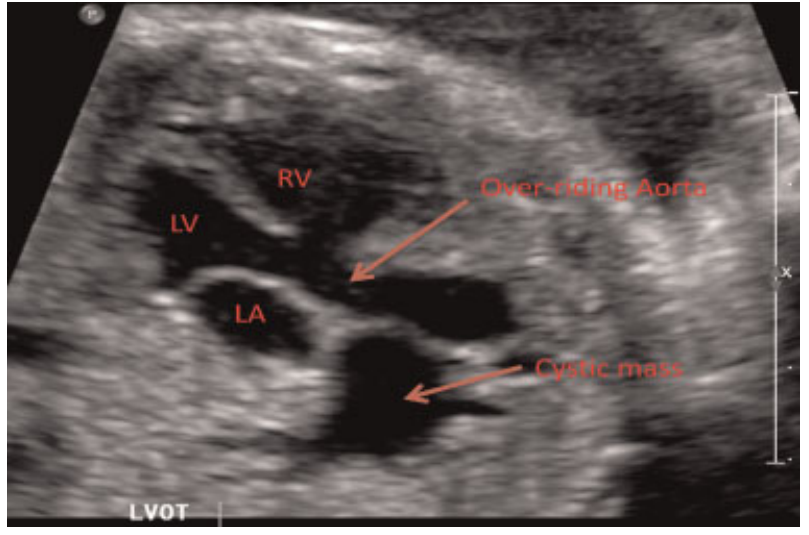

Figure 1 A fetal echocardiogram showing the aortic valve overriding a malalignment ventricular septal defect and a cystic mass above the left atrium (LA). LV, left ventricle; RV, right ventricle.

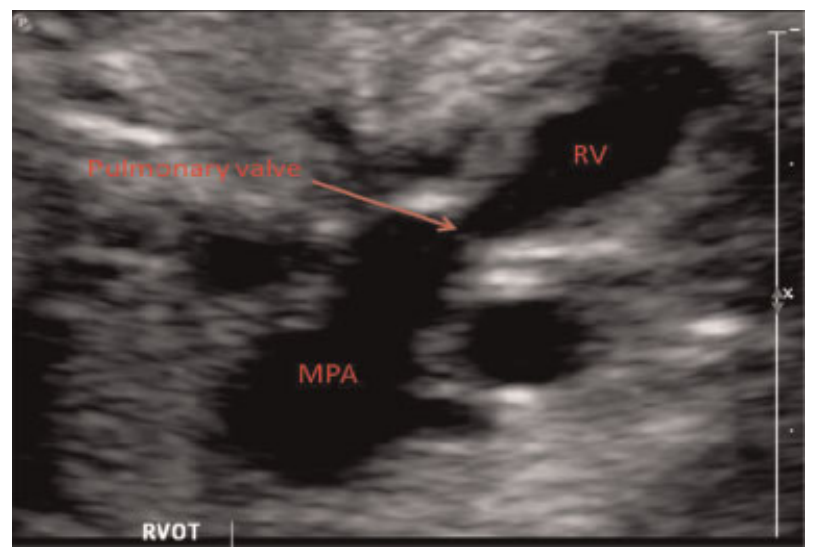

Figure 2 A fetal echocardiogram showing a stenotic pulmonary valve with absent leaflets and dilated main pulmonary artery (MPA). RV, right ventricle.

syndromes, and she declined genetic amniocentesis. She was followed with serial fetal echocardiograms, and no signs of fetal hydrops or heart failure were detected throughout the remainder of the pregnancy. At 38 weeks of gestation, a 3807-g female infant was born by a spontaneous vaginal delivery with Apgar scores of 8 and 9 at 1 and 5 minutes, respectively.

Physical examination of the neonate showed a welldeveloped full-term female infant without any dysmorphic features and no respiratory distress. Her oxygen saturation was $96 \%$ at room air. The cardiac examination was remarkable for a prominent right ventricular impulse and a single second heart sound. There was a classic to-and-fro murmur at the upper left sternal border. Genetic testing with microarray analysis did not detect any specific anomaly.

A neonatal echocardiogram confirmed the presence of TOF/APVS. There was a secundum atrial septal defect (ASD) and a right aortic arch. The pulmonary valve annulus was hypoplastic $(3.5 \mathrm{~mm})$ and the aortic valve annulus was $9.8 \mathrm{~mm}$. The MPA and RPA were dilated and measured $19 \mathrm{~mm}$ and $16 \mathrm{~mm}$, respectively. The LPA was diffusely hypoplastic $(3.9 \mathrm{~mm})$ and originated from the posterior aspect of the ascending aorta ( $\mathbf{- F i g . ~ 3 ) . ~ T h e r e ~ w a s ~ m i l d ~}$ pulmonary valve stenosis (peak velocity $2.7 \mathrm{~m} / \mathrm{s}$ ) and severe regurgitation. No PDA was detected.

The neonate underwent surgical repair on day 13 of life. The surgery consisted of mobilization of the LPA, reduction angioplasty of the MPA and RPA, patch closure of the VSD and ASD. A homograft was placed between the right ventricle and the MPA. The patient did remarkably well postoperatively and stayed in the hospital for 1 week. In her last clinic visit at 10 months of age, she was doing clinically well, but an echocardiogram showed a mild diffuse narrowing of the LPA and a mild homograft stenosis.

\section{Discussion}

Prenatal diagnosis of APVS has been reported as early as 22 weeks of gestation. ${ }^{5}$ Nearly one-fourth of the cases TOF/ APVS are associated with other CHD such as dextrocardia, ${ }^{2}$ atrioventricular septal defects, ${ }^{3}$ and others. We report a novel association of TOF/APVS with an abnormal origin of the LPA from the ascending aorta (hemitruncus). This was suspected

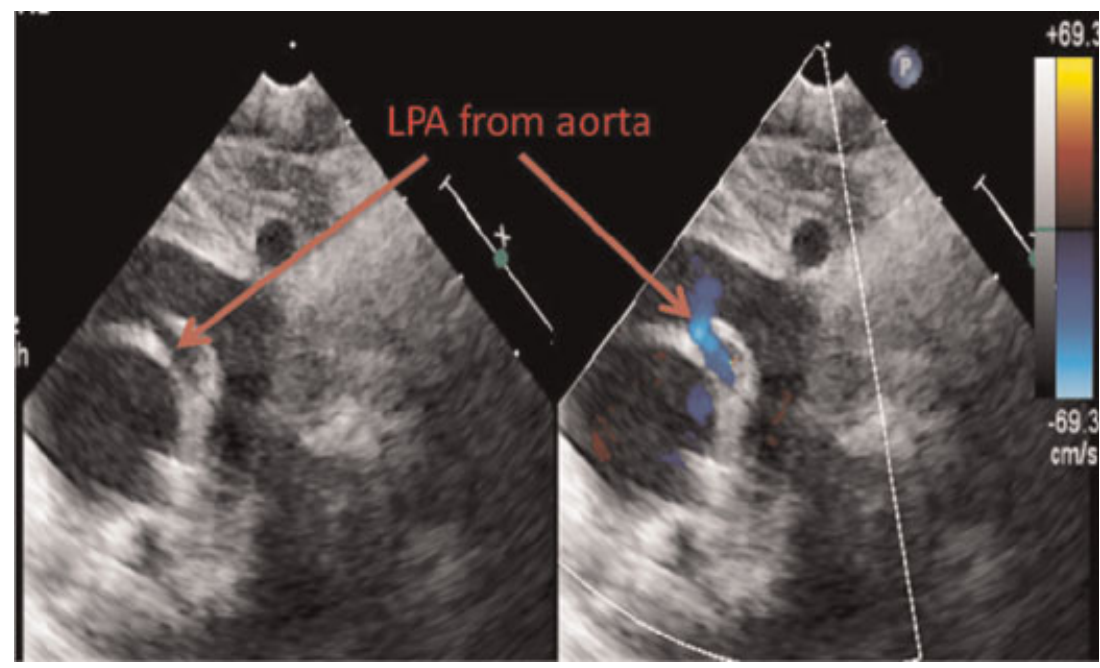

Figure 3 A postnatal 2-D and color Doppler echocardiogram of the aortic arch showing the abnormal origin of the left pulmonary artery (LPA) from the posterior aspect of the ascending aorta. 
antenatally on fetal echocardiography at 22 weeks' gestation and was confirmed postnatally. The patient underwent early surgical repair with a favorable outcome. In a literature search performed by the authors, this association has not been published in the English literature to date.

\section{References}

1 Galindo A, Gutiérrez-Larraya F, Martínez JM, et al. Prenatal diagnosis and outcome for fetuses with congenital absence of the pulmonary valve. Ultrasound Obstet Gynecol 2006;28:32-39

2 Bharati AH, Naware A, Merchant SA. Absent pulmonary valve syndrome with tetralogy of Fallot and associated dextrocardia detected at an early gestational age of 26 weeks. Indian J Radiol Imaging 2008;18:352-354

3 Kikuchi S, Yokozawa M. Absent pulmonary valve associated with tetralogy of Fallot and complete atrioventricular septal defect: report of a case. Ann Thorac Cardiovasc Surg 2005;11: 44-47

4 Yeager SB, Van Der Velde ME, Waters BL, Sanders SP. Prenatal role of the ductus arteriosus in absent pulmonary valve syndrome. Echocardiography 2002;19:489-493

5 Becker R, Schmitz L, Guschmann M, Wegner RD, Stiemer B, Entezami M. Prenatal diagnosis of familial absent pulmonary valve syndrome: case report and review of the literature. Ultrasound Obstet Gynecol 2001;17:263-267 